\title{
A Fast Passivity Test for Descriptor Systems Via Structure-Preserving Transformations of Skew-Hamiltonian/Hamiltonian Matrix Pencils
}

\author{
N. Wong \\ Department of Electrical and Electronic Engg \\ The University of Hong Kong \\ Pokfulam Road, Hong Kong \\ nwong@eee.hku.hk
}

\author{
C.K. Chu \\ Department of Electrical and Electronic Engg \\ The University of Hong Kong \\ Pokfulam Road, Hong Kong \\ ckchu@eee.hku.hk
}

\begin{abstract}
Passivity in a VLSI model is an important property to guarantee stable global simulation. Most VLSI models are naturally described as descriptor systems (DSs) or singular state spaces. Passivity tests for DSs, however, are much less developed compared to their nonsingular state space counterparts. For large-scale DSs, the existing test based on linear matrix inequality (LMI) is computationally prohibitive. Other system decoupling techniques involve complicated coding and sometimes ill-conditioned transformations. This paper proposes a simple DS passivity test based on the key insight that the sum of a passive system and its adjoint must be impulse-free. A sidetrack shows that the proper (non-impulsive) part of a passive DS can be easily decoupled along the test flow. Numerical examples confirm the effectiveness of the proposed DS passivity test over conventional approaches.
\end{abstract}

\section{Categories and Subject Descriptors}

I.6.5 [Simulation and Modeling]: Model Development-modeling methodologies; J.6 [Computer-Aided Engineering]: —computeraided design $(C A D)$

\section{General Terms}

Algorithms

\section{Keywords}

Descriptor system, positive real, passivity test

\section{INTRODUCTION}

Descriptor systems (DSs) have been widely used in modelings such as robotics [1], micro-electromechanical systems (MEMS) [2], and especially VLSI circuit simulations [3,4]. Many circuit modeling techniques, such as modified nodal analysis (MNA) $[5,6]$, naturally produce models in DS format. On the other hand, passiv-

Permission to make digital or hard copies of all or part of this work for personal or classroom use is granted without fee provided that copies are not made or distributed for profit or commercial advantage and that copies bear this notice and the full citation on the first page. To copy otherwise, to republish, to post on servers or to redistribute to lists, requires prior specific permission and/or a fee.

DAC 2006, July 24-28, 2006, San Francisco, California, USA

Copyright 2006 ACM 1-59593-381-6/06/0007 ...\$5.00. ity $^{1}$ or positive realness in a VLSI model is an important property to guarantee stable global simulation [3,7]. Existing DS passivity tests are restrictive in different aspects. For example, the extended linear matrix inequality (LMI) test in [7] has a high complexity of $O\left(n^{5}\right)$ to $O\left(n^{6}\right)$, rendering it prohibitive in testing passivity of high-order DSs, as is usual for VLSI models. The generalized algebraic Riccati equation (GARE) test [8] works only in the limited case of admissible (regular, stable and impulse-free) DSs.

The contribution of this paper is the formulation of a fast $O\left(n^{3}\right)$ algorithm for checking passivity of a DS. The key insight is that when a (possibly impulsive) passive system is added to its adjoint, the resulting system, which is again a DS, must be impulse-free. Numerically efficient and reliable techniques in transforming skewHamiltonian/Hamiltonian (SHH) matrix pencils are employed. After removal of uncontrollable and unobservable impulsive modes, if any, passivity can then be checked through the positive semidefinite of the residue matrix and the proper part of the DS using standard techniques (e.g., [9,10]). Along the proposed test, numerically well-conditioned orthogonal transformations are used whenever possible. The proper part of the original DS is also conveniently decoupled as a sidetrack.

Section 2 of this paper reviews the problem settings and important DS properties. Linear system transformations necessary for the development of the proposed test are covered. Section 3 presents a walkthrough of the proposed DS passivity test under the framework of SHH matrix pencil transformations. Numerical results in Section 4 show the effectiveness of the proposed approach over conventional schemes. Finally, Section 5 draws the conclusion.

\section{BASICS OF DESCRIPTOR SYSTEMS}

This section reviews some basic DS properties [7,8,11-15] that enable formulation of the proposed passivity test. We consider a linear time-invariant continuous-time DS of the form

$$
\begin{aligned}
& E \dot{x}=A x+B u, E x\left(0_{-}\right)=E x_{0} \\
& y=C x+D u
\end{aligned}
$$

where $E, A \in \mathbb{R}^{n \times n}, B, C^{T} \in \mathbb{R}^{n \times m}, D \in \mathbb{R}^{m \times m}$, and $E$ is generally singular with $\operatorname{rank}(E)=r \leq n$. In dealing with passivity, the system is assumed to be square such that the quantity $u^{T} y$ is interpreted as the instantaneous power injected into the system. We assume a regular pencil $(E, A)$, i.e., $\exists s_{0} \in \mathbb{C}$,

\footnotetext{
${ }^{1}$ A passive system is one that does not generate energy. A strictly passive system is dissipative and is automatically stable.
} 
$\operatorname{det}\left(-s_{0} E+A\right) \neq 0$, such that the transfer function

$$
G(s)=D+C(s E-A)^{-1} B=:\left[\begin{array}{c|c}
-s E+A & B \\
\hline C & D
\end{array}\right]
$$

is uniquely determined. If the degree, $\operatorname{deg}(\operatorname{det}(-s E+A))=$ $q(\leq r)$, then $(E, A)$ has $q$ finite dynamic modes, $(n-r)$ nondynamic modes, and $(r-q)$ impulsive modes. A nondynamic mode corresponds to an infinite eigenvalue with a grade- 1 generalized eigenvectors $v^{(1)}$ that satisfies $E v^{(1)}=0$. The infinite eigenvalues corresponding to the generalized grade- $k$ vectors $v^{(k)}$ that satisfy $E v^{(k)}=A v^{(k-1)}, k \geq 2$, are the impulsive modes. When all the finite dynamic modes of $(E, A)$ are stable, then we say $(E, A)$ is stable. If $(E, A)$ is regular, stable, and impulse-free, then $(E, A)$ is termed admissible. For a linear time-invariant system, passivity is equivalent to positive realness of the transfer function.

\subsection{Passivity and Positive Realness}

A rational matrix-valued transfer function $G(s) \in \mathbb{R}^{m \times m}$ is positive real (strictly positive real) if

1. $G(s)$ is analytic in $\mathbb{C}_{+}=\{s \in \mathbb{C} \mid \operatorname{Re}(s)>0\}$.

2. $\Phi(j \omega)=G(j \omega)+(G(j \omega))^{*} \geq 0$ (>0 for strict positive realness), $\forall \omega \in \mathbb{R}$, with $j \omega$ not a pole of $G(s)$.

3. If $j \omega_{0}$ or $\infty$ is a pole of $G(s)$, then it is a simple pole and the $m \times m$ residue matrix or first Markov parameter is positive semidefinite.

If $G(s)$ is decomposed as

$$
G(s)=\overbrace{\underbrace{G_{s p}(s)}_{\text {strictly proper }}+M_{0}}^{=G_{p}(s) \text { proper part }}+s M_{1}+\sum_{k=2}^{\infty} s^{k} M_{k},
$$

then $G(s)$ is positive real if and only if $G_{p}(s)$ is positive real and $M_{1} \geq 0, M_{k}=0, k \geq 2$. In the rest of the paper, we assume $(E, A)$ is regular and stable (i.e., the eigenvalues $\lambda(E, A) \subset$ $\left.\left\{\mathbb{C}_{-} \cup \infty\right\}\right)$ as in the modeling of passive circuits. Therefore, the first condition in the positive realness test is always true. However, we make no assumption about the impulsive part of $G(s)$, namely, it may have nonzero Markov parameters.

\subsection{LMI Passivity Test for a DS}

Ref [7] presents the LMI passivity test for a DS: (Sufficiency) If the linear matrix inequalities (LMIs)

$$
\left[\begin{array}{cc}
A^{T} X+X^{T} A & X^{T} B-C^{T} \\
B^{T} X-C & -\left(D+D^{T}\right)
\end{array}\right] \leq 0, E^{T} X=X^{T} E \geq 0
$$

have a solution $X$, then $G(s)$ is positive real.

(Necessity) Suppose $(E, A, B, C, D)$ is a minimal realization of $G(s)$ with $D+D^{T} \geq M_{0}+M_{0}^{T}$ where $M_{0}$ is the zeroth Markov parameter of $G(s)$ as in (3). If $G(s)$ is positive real, then the LMIs in (4) have a solution $X$.

In a regular system, $E$ is nonsingular and can be assumed to be identity without loss of generality $\left(E^{-1}\right.$ can be absorbed into $A$ and $B$ ). The LMI tests in (4) then reduce to the famous LMI formulation of the positive real lemma for regular systems, e.g., [7]. Strict positive realness can be checked by the existence of a stabilizing solution $X=X^{T} \geq 0$ to the algebraic Riccati equation (ARE)

$$
A^{T} X+X A+\left(X B-C^{T}\right)\left(D+D^{T}\right)^{-1}\left(B^{T} X-C\right)=0
$$

where $D+D^{T}>0$ can be assumed without loss of generality for passive systems [16]. In fact, the technique in [16] can be extended to a recursive reduction algorithm so that this ARE-based test, of $O\left(n^{3}\right)$ work, can be applied for checking (non-strict) positive realness, too. The existence of the solution $X$ can then be inferred from the nonexistence of purely imaginary eigenvalue in an associated Hamiltonian matrix (see e.g., $[9,10]$, for details).

\subsection{Equivalent Transformations}

Assume compatible partitions and dimensions, the new DS tuple $\left(E^{\prime}, A^{\prime}, B^{\prime}, C^{\prime}, D^{\prime}\right)$ obtained from

$$
\begin{aligned}
& {\left[\begin{array}{c|c}
-s E^{\prime}+A^{\prime} & B^{\prime} \\
\hline C^{\prime} & D^{\prime}
\end{array}\right]:=} \\
& {\left[\begin{array}{cc}
Q & 0 \\
M & I
\end{array}\right]^{T}\left[\begin{array}{c|c}
-s E+A & B \\
\hline C & D
\end{array}\right]\left[\begin{array}{cc}
Z & 0 \\
R & I
\end{array}\right]}
\end{aligned}
$$

where $Q, Z$ are nonsingular and $M^{T} E=E R=0$, is called a strong equivalent (s.e.) transform. When $M=R=0$, it is called a restricted system equivalent (r.s.e.) transform [11]. It is routine to show that s.e. (and therefore r.s.e.) preserves the transfer function of the original DS. Obviously, r.s.e. is a generalization of the similarity transform in regular system theory, while s.e. is even more general with a $D^{\prime}$ (feedthrough) generally different from $D$.

\subsection{Singular Value Decomposition (SVD) and Weierstrass Coordinates}

Performing the singular value decomposition (SVD) of $E=$ $U\left[\begin{array}{cc}\Sigma & 0 \\ 0 & 0\end{array}\right] V^{T}$, where $\operatorname{rank}(\Sigma)=r$, the DS can be transformed by a simple r.s.e. into the SVD coordinate $[13,15]$

$$
\begin{gathered}
U^{T} E V=\left[\begin{array}{ll}
\Sigma & 0 \\
0 & 0
\end{array}\right] \quad, \quad U^{T} A V=\left[\begin{array}{ll}
A_{11} & A_{12} \\
A_{21} & A_{22}
\end{array}\right] \\
U^{T} B=\left[\begin{array}{l}
B_{1} \\
B_{2}
\end{array}\right] \quad, \quad C V=\left[\begin{array}{ll}
C_{1} & C_{2}
\end{array}\right] .
\end{gathered}
$$

In fact, in the context of this paper, $\Sigma$ can be relaxed to any nonsingular matrix $E_{11}$ without affecting the results. Another important r.s.e. system results from the Weierstrass form [7] of the matrix pencil $(E, A)$. Specifically, for a regular pencil $(E, A)$, there are nonsingular $Q$ and $Z$ such that

$$
\begin{gathered}
Q^{T} E Z=\left[\begin{array}{cc}
I_{q} & 0 \\
0 & N
\end{array}\right] \quad, \quad Q^{T} A Z=\left[\begin{array}{cc}
A_{p} & 0 \\
0 & I_{n-q}
\end{array}\right] \\
Q^{T} B=\left[\begin{array}{c}
B_{p} \\
B_{\infty}
\end{array}\right] \quad, \quad C Z=\left[\begin{array}{ll}
C_{p} & C_{\infty}
\end{array}\right],
\end{gathered}
$$

where $q=\operatorname{deg}(\operatorname{det}(-s E+A))$ as in the settings in (1), $A_{p} \in$ $\mathbb{R}^{q \times q}$ contains the finite modes of $G(s)$, and $N \in \mathbb{R}^{(n-q) \times(n-q)}$ is nilpotent, i.e., $N^{v}=0$ for some integer $v \geq 1$. Consequently, any $G(s)$ can be additively decomposed into

$$
\begin{aligned}
G(s) & =D+C_{p}\left(s I-A_{p}\right)^{-1} B_{p}+C_{\infty}(s N-I)^{-1} B_{\infty} \\
& =D+C_{p}\left(s I-A_{p}\right)^{-1} B_{p}-C_{\infty}\left(\sum_{k=0}^{v-1} s^{k} N^{k}\right) B_{\infty}
\end{aligned}
$$

corresponding to (3). However, it should be stressed that Weierstrass form is mainly used in theoretical proofs. Transformation to this form may involve ill-conditioned, non-orthogonal transformations and therefore not favorable for numerical implementations.

\subsection{Impulse Controllability and Observability}

This section provides characterizations of impulsive behavior in a DS. Some of them, e.g., the SVD tests, are convenient tests for 
impulsive modes but are less well-known. In the following, we put together these tests in a coherent manner for easy reference.

Impulse-Free Case: Suppose $u(t) \equiv 0$, the DS in (1) is impulsefree if all free responses $x(t)$ are smooth in the time interval $[0, \infty)$ for all admissible initial conditions $x_{0}$. Alternatively, there exists no free response of the form $x(t)=v \delta(t), 0 \neq v \in \mathbb{R}^{n}$. Moreover, the following statements are equivalent:

1. The pair $(E, A)$ is impulse-free;

2. If there exist $v, w \in \mathbb{R}^{n}$ such that $(s E-A) v=E w, \forall s \in$ $\mathbb{C}$, then $v=0$.

3. $\left(A^{-1} \operatorname{Im} E\right) \cap \operatorname{Ker} E=0$;

4. $\operatorname{Im} E^{T}+A^{T} \operatorname{Ker} E^{T}=\mathbb{R}^{n}$;

5. In SVD coordinate, $A_{22}$ either vanishes or has full rank (i.e., nonsingular).

Impulse Observability: Suppose $u(t) \equiv 0$, the DS in (1) is impulse observable if $y(t)$ is impulse free for $t \geq 0$, only if $x(t)$ is impulse free for $t \geq 0$. Alternatively, there exists no free response of the form $x(t)=v \delta(t), 0 \neq v \in \mathbb{R}^{n}$ such that $y(t)=0, t \geq 0$. Moreover, the following statements are equivalent:

1. The triple $(E, A, C)$ is impulse observable;

2. If there exist $v, w \in \mathbb{R}^{n}$ such that $(s E-A) v=E w$ and $C v=0, \forall s \in \mathbb{C}$, then $v=0$.

3. $\left(A^{-1} \operatorname{Im} E\right) \cap \operatorname{Ker} E \cap \operatorname{Ker} C=0$;

4. $\operatorname{Im} E^{T}+A^{T} \operatorname{Ker} E^{T}+\operatorname{Im} C^{T}=\mathbb{R}^{n}$;

5. In SVD coordinate, $\left[\begin{array}{c}A_{22} \\ C_{2}\end{array}\right]$ either vanishes or has full column rank.

Impulse Controllability: The DS in (1) is impulse controllable if for every admissible initial condition $x_{0}$ there exists a smooth (impulse-free) control $u(t)$ for $t \geq 0$ such that the state trajectory $x(t)$ is smooth for $t \geq 0$. Moreover, the following statements are equivalent:

1. The triple $(E, A, B)$ is impulse controllable;

2. If there exist $v, w \in \mathbb{R}^{n}$ such that $v^{T}(s E-A)=w^{T} E$ and $v^{T} B=0, \forall s \in \mathbb{C}$, then $v=0$.

3. $\left(A^{-T} \operatorname{Im} E^{T}\right) \cap \operatorname{Ker} E^{T} \cap \operatorname{Ker} B^{T}=0$;

4. $\operatorname{Im} E+A \operatorname{Ker} E+\operatorname{Im} B=\mathbb{R}^{n}$;

5. In SVD coordinate, $\left[\begin{array}{ll}A_{22} & B_{2}\end{array}\right]$ either vanishes or has full row rank.

\section{STRUCTURE-PRESERVING TEST FOR DS PASSIVITY}

This section presents a step-by-step walkthrough of the main results, namely, on a fast passivity test for DSs. Instead of former approaches that work on $G(s)$, we directly analyze $\Phi(s)=$ $G(s)+G^{\sim}(s)$ which is also a DS (of double the order). The idea stems from the fact that even with the presence of an impulsive part in $G(s)$, which constitutes most of the numerical difficulties in a DS passivity check, it will be canceled out when $G(s)$ is added to its adjoint $G^{\sim}(s)=G^{T}(-s)$, provided $G(s)$ is passive. Fig. 1 captures the flow of the proposed test. First, we form

$$
\Phi(s)=G(s)+G^{\sim}(s)=\left[\begin{array}{c|c}
-s E_{\phi}+A_{\phi} & J C_{\phi}^{T} \\
\hline C_{\phi} & D_{\phi}
\end{array}\right]
$$

where

$$
\begin{array}{ll}
E_{\phi}=\left[\begin{array}{cc}
E & 0 \\
0 & E^{T}
\end{array}\right], & A_{\phi}=\left[\begin{array}{cc}
A & 0 \\
0 & -A^{T}
\end{array}\right] \\
C_{\phi}=\left[\begin{array}{ll}
C & B^{T}
\end{array}\right], & D_{\phi}=D+D^{T}
\end{array}
$$

Here $J=\left[\begin{array}{cc}0 & I \\ -I & 0\end{array}\right]$ is of compatible dimension, and $\left(E_{\phi}, A_{\phi}\right)$ is a skew-Hamiltonian/Hamiltonian (SHH) matrix pencil [17]. Some quick facts of (skew-)Hamiltonian matrix necessary for later derivations are summarized here (see e.g., [17]):

1. A Hamiltonian matrix $H$ and a skew-Hamiltonian matrix $W$ look like

$$
H=\left[\begin{array}{cc}
\hat{A} & \hat{R} \\
\hat{Q} & -\hat{A}^{T}
\end{array}\right], W=\left[\begin{array}{cc}
\hat{F} & \hat{\Theta} \\
\hat{\Omega} & \hat{F}^{T}
\end{array}\right]
$$

where $\hat{R}=\hat{R}^{T}, \hat{Q}=\hat{Q}^{T}, \hat{\Theta}=-\hat{\Theta}^{T}$, and $\hat{\Omega}=-\hat{\Omega}^{T}$.

2. $(J H)^{T}=J H$ and $(J W)^{T}=-J W$. Eigenvalues of a Hamiltonian matrix occur in quadruplets $(\lambda, \bar{\lambda},-\lambda,-\bar{\lambda})$ or in real or purely imaginary pairs $(\lambda,-\lambda)$.

3. A nonsingular symplectic matrix $S$ satisfies $S^{T} J S=J$. An orthogonal symplectic matrix $S$ further has $S^{T} S=I$. Similarity transform of a (skew-)Hamiltonian matrix by $S$, i.e., $\left(S^{-1} W S\right) S^{-1} H S$, is still (skew-)Hamiltonian.

Referring to (3) and (10), if $G(s)$ is passive and has an impulsive part, then all impulsive modes in $\Phi(s)$ must become unobservable and uncontrollable, and thereby would not appear in the minimal realization of $\Phi(s)$. In other words, any impulsive mode in $\Phi(s)$ can be eliminated through a reduction process depicted below.

\subsection{Removing Unobservable and Uncontrol- lable Impulse Modes}

Referring to Section 2.5, an impulse unobservable mode (direction), if any, is given by a $z$ that satisfies

$$
z^{*}\left[\begin{array}{lll}
E_{\phi}^{T} & A_{\phi}^{T} \operatorname{Ker} E_{\phi}^{T} & C_{\phi}^{T}
\end{array}\right]=0
$$

with $(\circ)^{*}$ being conjugate transpose. Here we have slightly abused the notation by using $\operatorname{Ker} E_{\phi}^{T}$ to stand for a matrix with orthonormal basis vectors that span the kernel of $E_{\phi}^{T}$ (and similarly below), which can be readily obtained using SVD. For every $z$ in (11), there exists a dual impulse uncontrollable mode, $-J z$, since

$$
\begin{aligned}
& (-J z)^{*}\left[\begin{array}{lll}
E_{\phi} & A_{\phi} \operatorname{Ker} E_{\phi} & J C_{\phi}^{T}
\end{array}\right] \\
= & z^{*}\left[\begin{array}{lll}
J E_{\phi} & J A_{\phi} \operatorname{Ker} E_{\phi} & -C_{\phi}^{T}
\end{array}\right] \\
= & z^{*}\left[\begin{array}{lll}
E_{\phi}^{T} J & A_{\phi}^{T} \operatorname{Ker} E_{\phi}^{T} & -C_{\phi}^{T}
\end{array}\right]=0 .
\end{aligned}
$$

The converse follows similarly. Let the orthonormal basis $Z_{\bar{o}}$ be a collection of all $z$ 's that satisfy (11). Henceforth, using the convention that italic of a matrix denotes the subspace that it spans, namely, $\mathcal{Z}_{\bar{o}}=\operatorname{span}\left(Z_{\bar{o}}\right)$, we have the important result

$$
\begin{aligned}
& \mathcal{Z}_{\bar{o}} \text { is impulse unobservable } \\
\Leftrightarrow \quad & -J \mathcal{Z}_{\bar{o}} \text { is impulse uncontrollable. }
\end{aligned}
$$


Next, construct $Z=\left[\begin{array}{ll}Z_{\bar{o}} & Z_{o}\end{array}\right]$ where $Z_{o}=Z_{\bar{o}}^{\perp}$ (so $\mathcal{Z}_{o}=\mathcal{Z}_{\overline{\bar{o}}}^{\perp}$ ). Finding $Q_{o}^{T} A_{\phi} Z_{\bar{o}}=0$, i.e., $\mathcal{Q}_{o}=\left(A_{\phi} \mathcal{Z}_{\bar{o}}\right)^{\perp}$, and similarly construct the orthonormal matrix $Q=\left[\begin{array}{ll}Q_{\bar{o}} & Q_{o}\end{array}\right]$ where $Q_{\bar{o}}=Q_{o}^{\perp}$, it follows analogously from the results in $[12,18]$ that

$$
\begin{aligned}
\Phi(s) & =\left[\begin{array}{cc}
Q & 0 \\
0 & I
\end{array}\right]^{T}\left[\begin{array}{c|c}
-s E_{\phi}+A_{\phi} & J C_{\phi}^{T} \\
\hline C_{\phi} & D_{\phi}
\end{array}\right]\left[\begin{array}{cc}
Z & 0 \\
0 & I
\end{array}\right] \\
& =\left[\begin{array}{cc|c}
Q_{\bar{o}}^{T} A_{\phi} Z_{\bar{o}} & -s Q_{\bar{o}}^{T} E_{\phi} Z_{o}+Q_{\bar{o}}^{T} A_{\phi} Z_{o} & Q_{\bar{o}}^{T} J C_{\phi}^{T} \\
0 & -s Q_{o}^{T} E_{\phi} Z_{o}+Q_{o}^{T} A_{\phi} Z_{o} & Q_{o}^{T} J C_{\phi}^{T} \\
\hline C_{\phi} Z_{o} & D_{\phi}
\end{array}\right] \\
& =\left[\begin{array}{c|c}
-s Q_{o}^{T} E_{\phi} Z_{o}+Q_{o}^{T} A_{\phi} Z_{o} & Q_{o}^{T} J C_{\phi}^{T} \\
\hline C_{\phi} Z_{o} & D_{\phi}
\end{array}\right] .
\end{aligned}
$$

Noting $\Phi(s)=G(s)+G^{\sim}(s)$, we also have

$$
\begin{aligned}
& \Phi(s)=\Phi^{\sim}(s)= \\
& \left.\qquad \begin{array}{c|c}
-s\left(J Z_{o}\right)^{T} E_{\phi}\left(J Q_{o}\right)+\left(J Z_{o}\right)^{T} A_{\phi}\left(J Q_{o}\right) & \left(J Z_{o}\right)^{T} J C_{\phi}^{T} \\
\hline C_{\phi}\left(J Q_{o}\right) & D_{\phi}
\end{array}\right]
\end{aligned}
$$

Inspecting (14) and (15), a key insight is that $J Q_{o}\left(Q_{o}\right)$ contains the unobservable $Z_{\bar{o}}$ (uncontrollable $-J Z_{\bar{o}}$ ). To prove so, again, using italic to denote the span of a matrix, and noting $A_{\phi}$ and $E_{\phi}$ are Hamiltonian and skew-Hamiltonian, respectively, we have

$$
\begin{aligned}
J \mathcal{Q}_{o} & =J\left(A_{\phi} \mathcal{Z}_{\bar{o}}\right)^{\perp}=J A_{\phi}^{-T} \mathcal{Z}_{o} \\
& =J A_{\phi}^{-T}\left(\operatorname{Im} E_{\phi}^{T}+A_{\phi}^{T} \operatorname{Ker} E_{\phi}^{T}+\operatorname{Im} C_{\phi}^{T}\right) \\
& =A_{\phi}^{-1} \operatorname{Im} E_{\phi}+\operatorname{Ker} E_{\phi}+A_{\phi}^{-1} \operatorname{Im} J C_{\phi}^{T} .
\end{aligned}
$$

Because the unobservable impulsive subspace $\mathcal{Z}_{\bar{o}}$ lies in $\operatorname{Ker} E_{\phi} \cap$ $\left(A_{\phi}^{-1} \operatorname{Im} E_{\phi}\right) \cap \operatorname{Ker} C_{\phi}$ the statement follows. Subsequently, we take the right projection matrix to be $Z_{c o}$ where $\mathcal{Z}_{c o}=J \mathcal{Q}_{o} \backslash$ $\left(J \mathcal{Q}_{o} \cap \mathcal{Z}_{\bar{o}}\right)$, and the left projection matrix $-J Z_{c o}$ since $-J \mathcal{Z}_{c o}=$ $\mathcal{Q}_{o} \backslash\left(\mathcal{Q}_{o} \cap-J \mathcal{Z}_{\bar{o}}\right)$. Here ' $\backslash$ ' denotes subtraction of sets [18]. These orthogonal projection matrices are readily obtained via SVDs. Unobservable and uncontrollable impulsive modes are then removed in the reduced-order r.s.e.

$$
\begin{aligned}
& {\left[\begin{array}{c|c}
-s E_{\phi 1}+A_{\phi 1} & -C_{\phi 1}^{T} \\
\hline C_{\phi 1} & D_{\phi 1}
\end{array}\right]:=} \\
& \quad\left[\begin{array}{cc}
-J Z_{c o} & 0 \\
0 & I
\end{array}\right]^{T}\left[\begin{array}{c|c}
-s E_{\phi}+A_{\phi} & J C_{\phi}^{T} \\
\hline C_{\phi} & D_{\phi}
\end{array}\right]\left[\begin{array}{cc}
Z_{c o} & 0 \\
0 & I
\end{array}\right]
\end{aligned}
$$

Note that $\left(E_{\phi 1}, A_{\phi 1}\right)$ is a skew-symmetric/symmetric pencil. A (minimal) passive $G(s)$ contains at most grade-2 generalized eigenvectors [7]. In this case, one pass of the above reduction procedure would clear all impulsive modes and leave the DS (17) with only nondynamic modes, whose removal is illustrated below. Any remaining impulsive modes (easily checked by tests in Section 2.5), irrespective of their observability and controllability, indicate a nonpassive $G(s)$ and the test is finished.

\subsection{Removing Nondynamic Modes}

The new DS tuple $\left(E_{\phi 1}, A_{\phi 1},-C_{\phi 1}^{T}, C_{\phi 1}, D_{\phi 1}\right)$ in (17), in case of passive original system, must be impulse-free and can at most contain nondynamic modes. Since $E_{\phi 1}$ is skew-symmetric, it can be decomposed into $E_{\phi 1}=U\left[\begin{array}{cc}E_{11} & 0 \\ 0 & 0\end{array}\right] U^{T}$ where $U$ is unitary and $E_{11}$ nonsingular with $\operatorname{rank}\left(E_{11}\right)=\operatorname{rank}\left(E_{\phi 1}\right)$. Another r.s.e. brings

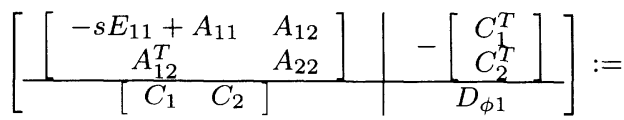

$$
\begin{aligned}
& {\left[\begin{array}{c|c}
-s U^{T} E_{\phi 1} U+U^{T} A_{\phi 1} U & -U^{T} C_{\phi 1}^{T} \\
\hline C_{\phi 1} U & D_{\phi 1}
\end{array}\right] .}
\end{aligned}
$$

Because $A_{22}$ is nonsingular for an impulse-free system, it is easily verified that (18) can be further reduced by a s.e. to

$$
\begin{aligned}
& {\left[\begin{array}{c|c}
-s E_{\phi 2}+A_{\phi 2} & -C_{\phi 2}^{T} \\
\hline C_{\phi 2} & D_{\phi 2}
\end{array}\right]:=} \\
& \quad\left[\begin{array}{c|c}
-s E_{11}+\left(A_{11}-A_{12} A_{22}^{-1} A_{12}^{T}\right) & -\left(C_{1}-C_{2} A_{22}^{-1} A_{12}^{T}\right)^{T} \\
\hline\left(C_{1}-C_{2} A_{22}^{-1} A_{12}^{T}\right) & \left(D_{\phi 1}-C_{2} A_{22}^{-1} C_{2}^{T}\right)
\end{array}\right]
\end{aligned}
$$

Next, a simple r.s.e. restores the $\mathrm{SHH}$ pencil in $\left(E_{\phi 3}, A_{\phi 3}\right)$

$$
\begin{aligned}
& {\left[\begin{array}{c|c}
-s E_{\phi 3}+A_{\phi 3} & J C_{\phi 3}^{T} \\
\hline C_{\phi 3} & D_{\phi 3}
\end{array}\right]:=} \\
& \quad\left[\begin{array}{c|c}
-s\left(-J E_{\phi 2}\right)+\left(-J A_{\phi 2}\right) & -J\left(-C_{\phi 2}^{T}\right) \\
\hline C_{\phi 2} & D_{\phi 2}
\end{array}\right]
\end{aligned}
$$

The DS in (20) is actually regular since $E_{\phi 3}=-J E_{11}$ is nonsingular and skew-Hamiltonian. This allows us to transform the system into a regular form for testing passivity of the proper part.

\subsection{Extracting the Stable Proper Part}

To start with, note that every skew-Hamiltonian matrix can be put into an upper block-triangular form using a simple $O\left(n^{3}\right)$ isotropic Arnoldi process [17]. This Arnoldi algorithm is easy to code and efficiently finds an orthogonal symplectic square matrix $Z$ such that $Z^{T} E_{\phi 3} Z=\left[\begin{array}{cc}F & \Theta \\ 0 & F^{T}\end{array}\right]$ where $\Theta^{T}=-\Theta$ is skew-symmetric. Let

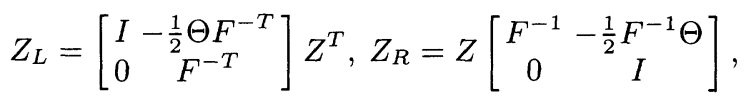

we get

$$
\begin{aligned}
\Phi(s) & =\left[\begin{array}{c|c}
-s I+A_{\phi 4} & J C_{\phi 4}^{T} \\
\hline C_{\phi 4} & D_{\phi 4}
\end{array}\right]:= \\
& {\left[\begin{array}{c|c}
-s Z_{L} E_{\phi 3} Z_{R}+Z_{L} A_{\phi 3} Z_{R} & Z_{L} J C_{\phi 3}^{T} \\
\hline C_{\phi 3} Z_{R} & D_{\phi 3}
\end{array}\right] . }
\end{aligned}
$$

To block-diagonalize $A_{\phi 4}$, which is a Hamiltonian matrix, and subsequently transform (21) into the sum of a proper system and its adjoint, assume $A_{\phi 4}=\left[\begin{array}{cc}\hat{A} & \hat{R} \\ \hat{Q} & -\hat{A}^{T}\end{array}\right]$. By assumption all finite poles of $G(s)$ are stable, $\Phi(s)$ has no pole on the imaginary axis and therefore the spectrum of $A_{\phi 4}$ can be equally divided into stable and unstable parts. If the dimension of $A_{\phi 4}$ is $2 n_{p} \times 2 n_{p}$, we can always find $\left[\begin{array}{ll}X_{1}^{T} & X_{2}^{T}\end{array}\right]^{T} \in \mathbb{R}^{2 n_{p} \times n_{p}}$ and $\Lambda \in \mathbb{R}^{n_{p} \times n_{p}}$ such that

$$
\left[\begin{array}{cc}
\hat{A} & \hat{R} \\
\hat{Q} & -\hat{A}^{T}
\end{array}\right]\left[\begin{array}{l}
X_{1} \\
X_{2}
\end{array}\right]=\left[\begin{array}{l}
X_{1} \\
X_{2}
\end{array}\right] \Lambda
$$

with $\operatorname{spec}(\Lambda)=\operatorname{spec}\left(A_{\phi 4}\right) \cap \mathbb{C}_{-}$. Without loss of generality, the stable invariant subspace $\left[\begin{array}{ll}X_{1}^{T} & X_{2}^{T}\end{array}\right]^{T}$ is assumed orthonormal (otherwise a $\mathrm{QR}$ factorization can do the task). It can be seen that $X_{1}^{T} X_{2}=X_{2}^{T} X_{1}[10]$ and $Z_{1}=\left[\begin{array}{cc}X_{1} & -X_{2} \\ X_{2} & X_{1}\end{array}\right]$ is orthogonal symplectic. Also,

$$
Z_{1}^{T}\left[\begin{array}{cc}
\hat{A} & \hat{R} \\
\hat{Q} & -\hat{A}^{T}
\end{array}\right] Z_{1}=\left[\begin{array}{cc}
\Lambda & \Psi \\
0 & -\Lambda^{T}
\end{array}\right] .
$$

Next, solving the Lyapunov equation $\Lambda Y+Y \Lambda^{T}+\Psi=0$ for $Y$ and forming $Z_{2}=Z_{1}\left[\begin{array}{ll}I & Y \\ 0 & I\end{array}\right]$ (thus $Z_{2}^{-1}=\left[\begin{array}{cc}I & -Y \\ 0 & I\end{array}\right] Z_{1}^{T}$ ), a 
similarity transformation gives

$$
\begin{gathered}
\Phi(s)=\left[\begin{array}{c|c}
-s I+A_{\phi 5} & J C_{\phi 5}^{T} \\
\hline C_{\phi 5} & D_{\phi 5}
\end{array}\right]:= \\
{\left[\begin{array}{c|c}
-s I+Z_{2}^{-1} A_{\phi 4} Z_{2} & Z_{2}^{-1} J C_{\phi 4}^{T} \\
\hline C_{\phi 4} Z_{2} & D_{\phi 4}
\end{array}\right]}
\end{gathered}
$$

where $A_{\phi 5}=\left[\begin{array}{cc}\Lambda & 0 \\ 0 & -\Lambda^{T}\end{array}\right]$. Recall that $\Phi(s)$ is preserved all the way through as we use only r.s.e. and s.e. transformations. Now it is readily seen that (23) represents the addition of a stable proper system and its adjoint.

\subsection{Extracting $\mathrm{M}_{1}$}

We also need to account for the positive semidefiniteness of any $M_{1}$, i.e., the impulsive part of $G(s)$, that may arise. Referring to (3), a symmetric $M_{2 k+1}$ or a skew-symmetric $M_{2 k}$ in $G(s)$, $k=1,2, \cdots$, will also result in uncontrollable or unobservable impulsive modes in $\Phi(s)$ in addition to those from a symmetric $M_{1}$ (if any). Fortunately, identification of these nonzero $M_{k}$ 's, and therefore a nonpassive $G(s)$, is simple by noting that a nonzero $M_{k}$ $(k \geq 1)$ is associated with nonzero grade- 1 to grade- $(k+1)$ generalized eigenvectors. Using the Weierstrass form or otherwise, it can be seen that $M_{1} \neq 0$ and $M_{k}=0, k \geq 2$, only when the total number of removed unobservable and uncontrollable modes in $\Phi(s)$ equals the total number of removed nondynamic modes. Here we give a simple way to extract $M_{1}$. Because minimal realization is assumed in the initial $G(s)$, every impulsive mode in $G(s)$ must be both controllable and observable. When only $M_{1}=M_{1}^{T}$ is present, we have equal numbers of grade- 1 and grade- 2 (and no higher-grade) generalized eigenvectors in the observable and controllable directions, respectively. Let $V_{o}^{(1)}\left(V_{c}^{(1)}\right)$ be the collection of all observable (controllable) grade- 1 generalized eigenvectors, there is an associated grade-2 eigenvector matrix $V_{o}^{(2)}\left(V_{c}^{(2)}\right)$ of full column rank such that

$$
\left\{\begin{array}{c}
E V_{o}^{(1)}=0 \\
E V_{o}^{(2)}=A V_{o}^{(1)}
\end{array} \quad, \quad\left\{\begin{array}{c}
E^{T} V_{c}^{(1)}=0 \\
E^{T} V_{c}^{(2)}=A^{T} V_{c}^{(1)}
\end{array} .\right.\right.
$$

The impulsive part (in this case $M_{1}$ only) of $G(s)$, denoted by the subscript " $\infty$ ", can then be extracted through the deflating subspace projection matrices $Z_{L \infty}=\left[\begin{array}{ll}V_{c}^{(1)} & V_{c}^{(2)}\end{array}\right]^{T}$ and $Z_{R \infty}=$ $\left[\begin{array}{ll}V_{o}^{(1)} & V_{o}^{(2)}\end{array}\right]$. Specifically, by forming $\left(E_{\infty}, A_{\infty}, B_{\infty}, C_{\infty}\right)=$ $\left(Z_{L \infty} E Z_{R \infty}, Z_{L \infty} A Z_{R \infty}, Z_{L \infty} B, C Z_{R \infty}\right)$, we have

$$
\begin{aligned}
M_{1} & =-C_{\infty} A_{\infty}^{-1} E_{\infty} A_{\infty}^{-1} B_{\infty} \\
& =C V_{o}^{(1)}\left(\left(V_{c}^{(1)}\right)^{T} A E^{\dagger} A V_{o}^{(1)}\right)^{-1}\left(V_{c}^{(1)}\right)^{T} B
\end{aligned}
$$

wherein $E^{\dagger}$ stands for the Moore-Penrose pseudo inverse of $E$. Invertibility of $A_{\infty}$ follows from nonsingularity of $\left(V_{c}^{(2)}\right)^{T} E V_{o}^{(2)}$, which is obvious from the Weierstrass forms of matrices. If $M_{1}$ is nonzero but not positive semidefinite, then $G(s)$ is not passive.

In other words, starting with an initial passive DS, we have constructively derived a way of identifying the proper and impulsive parts. Testing passivity of the original system is then equivalent to testing the passivity of these extracted parts. If the transformation and reduction fail somewhere in the flow in Fig. 1, then it can be concluded that the initial DS is not passive.

\section{NUMERICAL EXPERIMENTS}

The proposed DS passivity test is contrasted with two conventional methods, namely, the extended LMI test in [7] and the Weierstrass approach wherein the proper and impulsive parts are first

\begin{tabular}{c||c|c|c}
$\begin{array}{c}\text { Model } \\
\text { order }\end{array}$ & $\begin{array}{c}\text { LMI } \\
\text { Test }\end{array}$ & $\begin{array}{c}\text { Proposed } \\
\text { method }\end{array}$ & $\begin{array}{c}\text { Weierstrass } \\
\text { decomposition }\end{array}$ \\
\hline \hline 20 & 5.633 & 0.1328 & 0.0859 \\
40 & 144.18 & 0.1875 & 0.1407 \\
60 & 1550.25 & 0.3047 & 0.2578 \\
80 & NIL & 0.5547 & 0.5136 \\
100 & NIL & 0.9922 & 1.0078 \\
200 & NIL & 14.7891 & 15.285 \\
400 & NIL & 155.1875 & 185.016 \\
\hline \hline
\end{tabular}

Table 1: CPU times (sec) for different passivity tests.

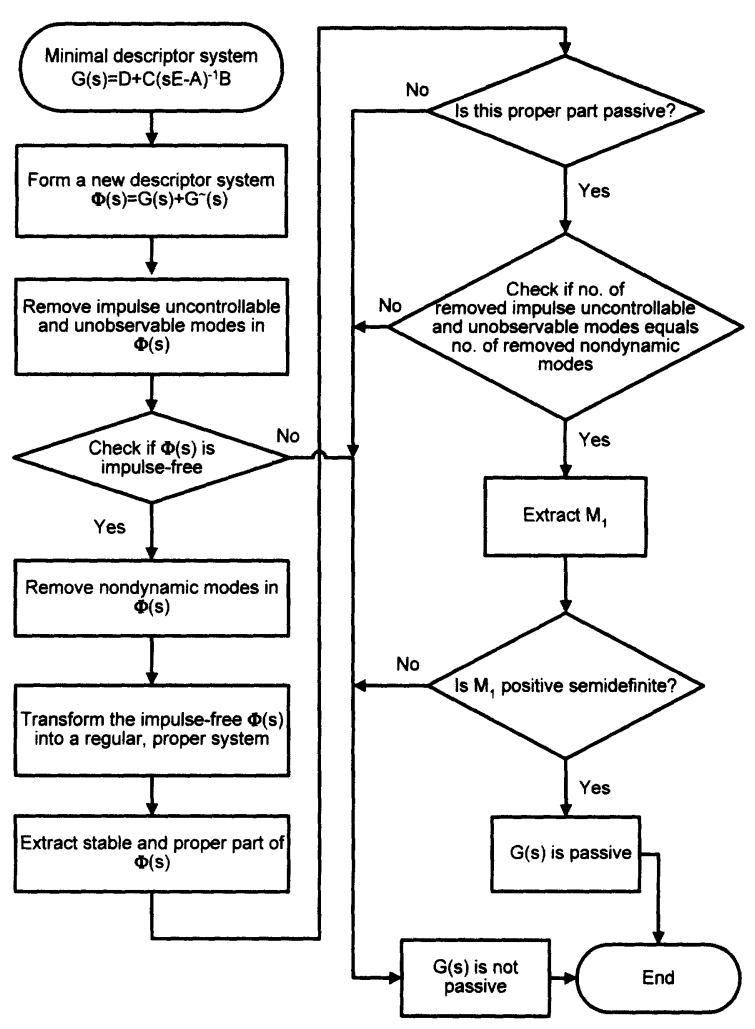

Figure 1: Flow of passivity test by proposed method.

separated and tested for positive realness (c.f. Sections 2.2 and 2.4). The algorithms are coded in Matlab 7.0.4 and executed on a $2.8 \mathrm{GHz}$ PC with $2 \mathrm{G}$ memory. Formation of Weierstrass form of matrix pencil $(-s E+A)$ is done by an algorithm called GUPTRI [19]. The CPU times of different passivity tests on practical RLC circuit models of different orders and number of impulsive modes are shown in Table 1 and plotted in Fig. 2, respectively. Referring to the table, the proposed passivity test and Weierstrass approach are both much faster than the LMI test. When model order is increased to 70, the LMI test fails due to physical memory limitations. When the model order is higher than 100 , the proposed approach also exhibits faster CPU times than the Weierstrass approach. Moreover, we note that though the Weierstrass test has a comparable speed to the proposed test (both of which are of $O\left(n^{3}\right)$ ), the former should be avoided in practice since it generally involves ill-conditioned and non-orthogonal transformations.

Some remarks are in order. First, the bottleneck of the proposed test is in the identification of stable invariant subspace in (22). We 

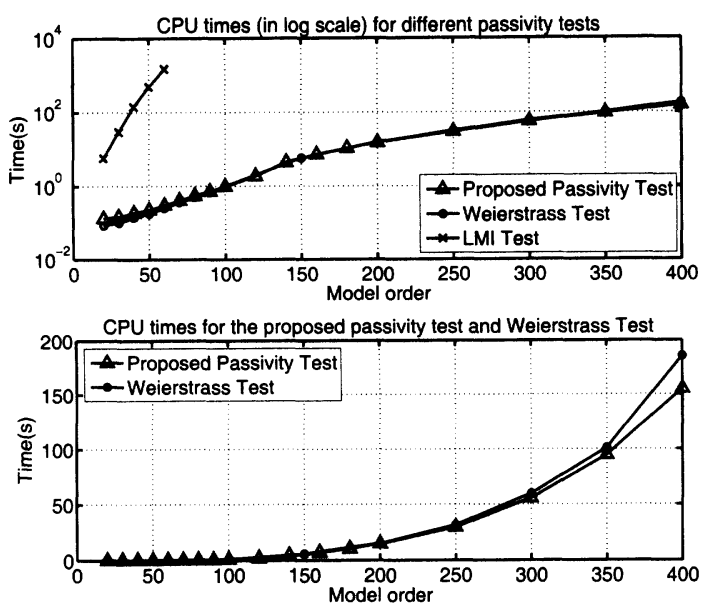

Figure 2: CPU times for different passivity tests.

are aware of a recent $O\left(n^{3}\right)$ DS passivity test described in [20]. However, due to the lack of implementation details the test has not been included here. In this regard, we note that the approach in [20] relies on iteratively transforming the DS matrix pencil (as in the Weierstrass approach) to a Schur form, followed by separate tests on the proper part and Markov parameters. The proposed test in this paper differs in the way that it is based on the self-cancellation of impulsive modes when a passive system is added to its adjoint. All transformation matrices are also formed in a constructive manner and therefore the computation is expected to be lower than a separation-based algorithm. Finally, the SHH matrix pencil reduction process developed for the passivity test also constitutes an effective way for delineating the proper part of a passive DS. Further applications such as passivity enforcement and DS model order reduction can readily be developed on top of this framework.

\section{CONCLUSION}

This paper has presented an efficient way for checking passivity of a possibly impulsive descriptor system (DS). Based on the self-cancellation of impulsive modes when a passive DS is added to its adjoint, a fast $O\left(n^{3}\right)$ algorithm is developed using skewHamiltonian/Hamiltonian ( $\mathrm{SHH}$ ) matrix pencil transformations. The proper part of the DS is also conveniently extracted as a sidetrack. Numerical examples have confirmed the effectiveness of the proposed DS passivity test over conventional methods.

\section{ACKNOWLEDGMENT}

This work is supported in part by the Hong Kong Research Grants Council under Project HKU 7173/04E and in part by the University Research Committee of The University of Hong Kong.

\section{REFERENCES}

[1] J. K. Mills and A. A. Goldenberg, "Force and position control of manipulators during constrained motion tasks," IEEE Trans. Robot. Automat., vol. 5, no. 1, pp. 30-46, Feb. 1989.

[2] E. B. Rudnyi and J. G. Korvink, "Model order reduction of mems for efficient computer aided design and system simulation," in Proc. Intl. Symp. Math. Theory of Networks and Systems, July 2004.
[3] Z. Bai, P. M. Dewilde, and R. W. Freund, "Reduced-order modeling," Numerical Analysis Manuscript No. 02-4-13, Bell Laboratories, Mar. 2002.

[4] J. R. Phillips, L. Daniel, and L. M. Silveira, "Guaranteed passive balancing transformations for model order reduction," IEEE Trans. Computer-Aided Design, vol. 22, no. 8, pp. 1027-1041, Aug. 2003.

[5] C. C. Chu, H. J. Lee, W. S. Feng, and M. H. Lai, "Interconnect model reductions by using the AORA algorithm with considering the adjoint network," in Proc. IEEE Intl. Symp. Circuits and Systems, vol. 2, may 2005, pp. 1278-1281.

[6] F. Y. Chang, "Unified nodal analysis of electronic circuits," in Proc. IEEE 39th Midwest Symp. Circuits and Systems, vol. 3, Aug. 1996, pp. 1119-1122.

[7] R. W. Freund and F. Jarre, "An extension of the positive real lemma to descriptor systems," Optimization Methods and Software, vol. 19, no. 1, pp. 69-87, Feb. 2004.

[8] L. Zhang, J. Lam, and S. Xu, "On positive realness of descriptor systems," IEEE Trans. Circuits Syst. I, vol. 49, no. 3, Mar. 2002.

[9] S. Grivet-Talocia, "Passivity enforcement via perturbation of hamiltonian matrices," IEEE Trans. Circuits Syst. I, vol. 51, no. 9, pp. 1755-1769, Sept. 2004.

[10] K. Zhou, J. C. Doyle, and K. Glover, Robust and Optimal Control. Upper Saddle River, NJ: Prentice-Hall, 1996.

[11] G. Verghese, B. C. Levy, and T. Kailath, "A generalized state-space for singular systems," IEEE Trans. Automat. Contr., vol. 26, no. 4, pp. 811-831, Aug. 1981.

[12] P. M. Van Dooren, "The generalized eigenstructure problem in linear system theory," IEEE Trans. Automat. Contr., vol. 26, no. 1, pp. 111-129, Feb. 1981.

[13] D. J. Bender and A. J. Laub, "The linear-quadratic optimal regulator for descriptor systems," IEEE Trans. Automat. Contr., vol. 32, no. 8, pp. 672-688, Aug. 1987.

[14] T. Katayama and K. Minamino, "Linear quadratic regulator and spectral factorization for continuous-time descriptor systems," in Proc. IEEE Conf. Decision and Control, 1992, pp. 967-972.

[15] J. Y. Ishihara and M. H. Terra, "Impulse controllability and observability of rectangular descriptor systems," IEEE Trans. Automat. Contr., vol. 46, no. 6, pp. 991-994, June 2001.

[16] H. Weiss, Q. Wang, and J. L. Speyer, "System characterization of positive real conditions," IEEE Trans. Automat. Contr., vol. 39, no. 3, pp. 540-544, Mar. 1994.

[17] V. Mehrmann and D. Watkins, "Structure-preserving methods for computing eigenpairs of large sparse skew-Hamiltonian/Hamiltonian pencils," SIAM J. Sci. Comput., vol. 22, no. 6, pp. 1905-1925, 2001.

[18] G. Basile and G. Marro, Controlled and Conditioned Invariants in Linear System Theory. Prentice Hall, 1992.

[19] J. Demmel and B. Kagstrom, "The generalized schur decomposition of an arbitrary pencil A- $\lambda \mathrm{B}$ : Robust software with error bounds and applications Part II: Software and application," ACM Transactions on Mathematical Software, vol. 19, no. 2, pp. 175-201, June 1993.

[20] P. Benner and D. Chu, "A new test for passivity of descriptor systems," Oberwolfach reports, Mathematisches Forschungsinstitut Oberwolfach, Tech. Rep. 11/2005, Feb 2005. 